\title{
Structural Optimization of Logistics Distribution System Based on Characteristic State Space
}

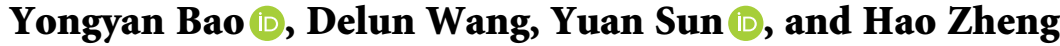 \\ School of Mechanical Engineering, Dalian University of Technology, Dalian 116024, China \\ Correspondence should be addressed to Yongyan Bao; baoyongyan@yeah.net
}

Received 4 November 2019; Accepted 8 January 2020; Published 25 February 2020

Academic Editor: Yuchuan Du

Copyright $\odot 2020$ Yongyan Bao et al. This is an open access article distributed under the Creative Commons Attribution License, which permits unrestricted use, distribution, and reproduction in any medium, provided the original work is properly cited.

\begin{abstract}
In this paper, resource flow variables are extracted from the internal structural features of the logistics distribution process and a new method for optimizing the internal structure of the logistics distribution system by using the characteristic state space is proposed. The characteristic state equation is constructed to represent the input and output resources of each basic logistics activity. The basic logistics activity equation is iterated according to the resource flow, and the implementation of the basic logistics process is visually and quantitatively expressed in the form of the characteristic state matrix. According to the nature of the characteristic state space, the optimization problem of the logistics distribution system is transformed into a critical path-planning problem, the gradient calculation of the objective function is solved, and an improved genetic algorithm is proposed. This accelerates the convergence speed of the algorithm and reduces the running time of the optimization process. Taking a listed logistics distribution enterprise as an example, the optimization algorithm is verified, which proves the advantages of the algorithm and provides a new method and theoretical basis for the analysis and optimization of the logistics distribution system.
\end{abstract}

\section{Introduction}

The modern logistics distribution process is the key link of the whole supply chain system construction. The distribution efficiency, cargo damage rate, and comprehensive logistics cost of the supply chain system are directly reflected in the logistics distribution process. The traditional logistics distribution mode mostly takes labor as the core factor. It will lead to high cost and low efficiency, and the cargo breakage rate is high, the overall profit margin of the industry is low, and the possibility of business interruption is increased.

With the rapid development of e-commerce and the popularity of "Internet + ," the logistics distribution industry has developed rapidly. For this reason, many scholars have carried out research from different angles. Rubtsov et al. proposed an approach to solve the problems of structural optimization and a set of calculation tasks, based on customer orders for analysis of the enterprise structure and infrastructure of the entire logistics system [1]; Tang and Veelenturf proposed the strategic of logistics in the industry 4.0 era [2]; Giusti et al. proposed synchromodal logistics: an overview of critical success factors, enabling technologies, and open research issues [3]. Khorshidian et al. proposed a hybrid intelligent product portfolio optimization model for solving the biobjective model [4]. Wieczorek et al. proposed continuous-domain genetic algorithms (GAs) for solving the resource distribution control in logistic systems [5]. Hsu et al. proposed a two-stage stochastic programming model for the optimization of logistics processes in modular construction [6]. Dymowa and Sevastjanov proposed a new method for crisp and fuzzy interval comparison based on the probabilistic approach for fuzzy simulation in logistic systems [7]. Gómez S. et al. proposed a method of Extended Constrained List (ECL) heuristics for solving a logistics system for the distribution of bottled products [8]. The above research is conducted mainly through optimization analysis and with a simulation model, setting constraints from a macroperspective to solve specific problems. From the internal structure of the system, we use a single variable to carry out research. At the same 
time, a lot of historical data are required. When analyzing complex logistics system problems, the solution process is time-consuming and laborious, especially the lack of the effective objective function gradient analysis method. This paper analyzes and optimizes the process of logistics distribution based on the algorithm of characteristic state space model developed by Professor Wang et al. [9-11]. Firstly, it describes the state characteristics of logistics distribution process by extracting the characteristic state vector. Secondly, we construct the characteristic state equation from the basic logistics process and directly and quantitatively express the basic logistics distribution process in the form of characteristic state matrix. Thirdly, a matrix decomposition and matching procedure can be performed in the model space to establish the overall logistics distribution process model, thus effectively revealing the relationship between the internal state of the complex logistics distribution system and the external input and output variables. The system state can then be described with the minimum information of the present and the past states, which is more time-saving and laborsaving.

\section{Basic Logistics Activity Model}

The logistics distribution process usually includes several basic operations such as purchasing, handling, storage, inventory, order processing, picking, replenishment, shipping, and distribution. For this basic logistics process, activities can be defined as follows:

Definition 1. A distribution batch of items A in a continuous delivery activity in a distribution center is called a basic logistics activity. This definition can be seen in the process of converting a batch of input resources (objects and information) into another batch of output resources, as shown in Figure 1(a). It is expressed by a matrix equation, such as formula (1). The basic logistics activity can be abstracted from the information flow angle to the vector. The transformation relationship between them, as shown in Figure 1(b), represents the state with a circular node and represents the transformation relationship between states with a directed arc. The matrix equation is expressed as follows:

$$
x_{o}=A x_{i}
$$

Definition 2. The multidimensional vector consisting of the values of the characteristic variables in different states before and after the completion of the basic logistics activities is called the input feature state vector and the output feature state vector of the basic logistics activities. A matrix describing the state transition relationship between input and output feature state vectors is called a feature transformation matrix. The matrix equation of formula (1) is composed of the feature state vector and the feature transformation matrix and is called the characteristic state equation of the basic logistics activity.

Formula (1) can be expressed in matrix form as

$$
\begin{aligned}
& \mathbf{x}_{i}=\left[\begin{array}{c}
t_{i} \\
q_{i} \\
c_{i}
\end{array}\right], \\
& \mathbf{x}_{o}=\left[\begin{array}{c}
t_{o} \\
q_{o} \\
c_{o}
\end{array}\right], \\
& \mathbf{A}=\left[\begin{array}{lll}
t_{p} & 0 & 0 \\
0 & q_{p} & 0 \\
0 & 0 & c_{p}
\end{array}\right],
\end{aligned}
$$

where $t_{i}, q_{i}, c_{i}, t_{o}, q_{o}, c_{o}, t_{p}, q_{p}$, and $c_{p}$, respectively, indicate the start time of the basic logistics activity, the quantity of purchase, procurement logistics cost, end time of basic logistics activities, shipment quantity, shipping logistics cost, delivery time of basic logistics activities, the number of damaged items (In terms of negative numbers), and the integrated logistics cost. The matrix elements represented by 0 indicate that there is no direct relationship between the corresponding feature variables.

\section{State Connection between Basic Logistics Activities}

In the actual logistics distribution process, each distribution process for each object requires a basic logistics processing activity. Therefore, the overall logistics distribution process for multiple items and multiple batch operations in a period is composed of multiple basic logistics activities. Then, it is necessary to further discuss the relationship between the articulated form of basic logistics activities and the operational equations of characteristic state equations.

3.1. Typical Distribution Process of Modern Logistics Enterprises. The logistics distribution enterprises belong to the discrete service industry. This paper investigates 11 modern logistics distribution enterprises and fully explores the common features and development status of modern logistics distribution links. We use FlexSim software [12] to construct the overall conceptual operation of the logistics distribution process. As shown in Figure 2, the overall logistics distribution center is divided into five districts and the five-zone logistics distribution concept operation diagram is drawn. The distribution process in the warehousing treatment area is taken as an example, and other districts are studied accordingly.

The main work of the object warehousing treatment is that of unloading objects, inspection, and transmission, and realizing the transshipment of objects outside the warehouse. The work center utilizes AGV transport trolleys or forklifts. When receiving the goods, if it is found that there are defective products, the goods cannot be put into the warehouse and they are sent to the temporarily storage area. 


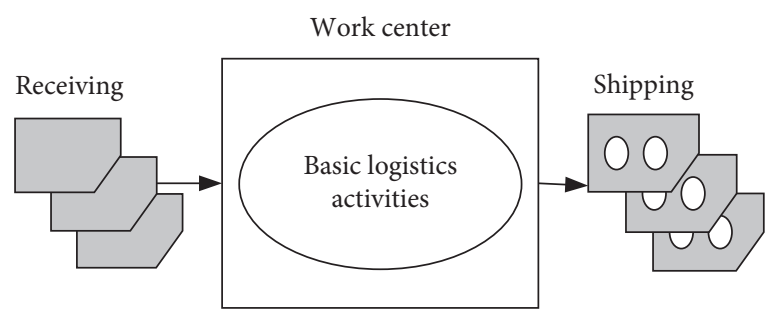

(a)

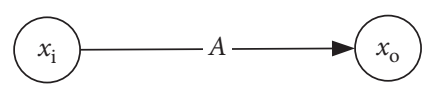

(b)

Figure 1: Basic logistics activity model.

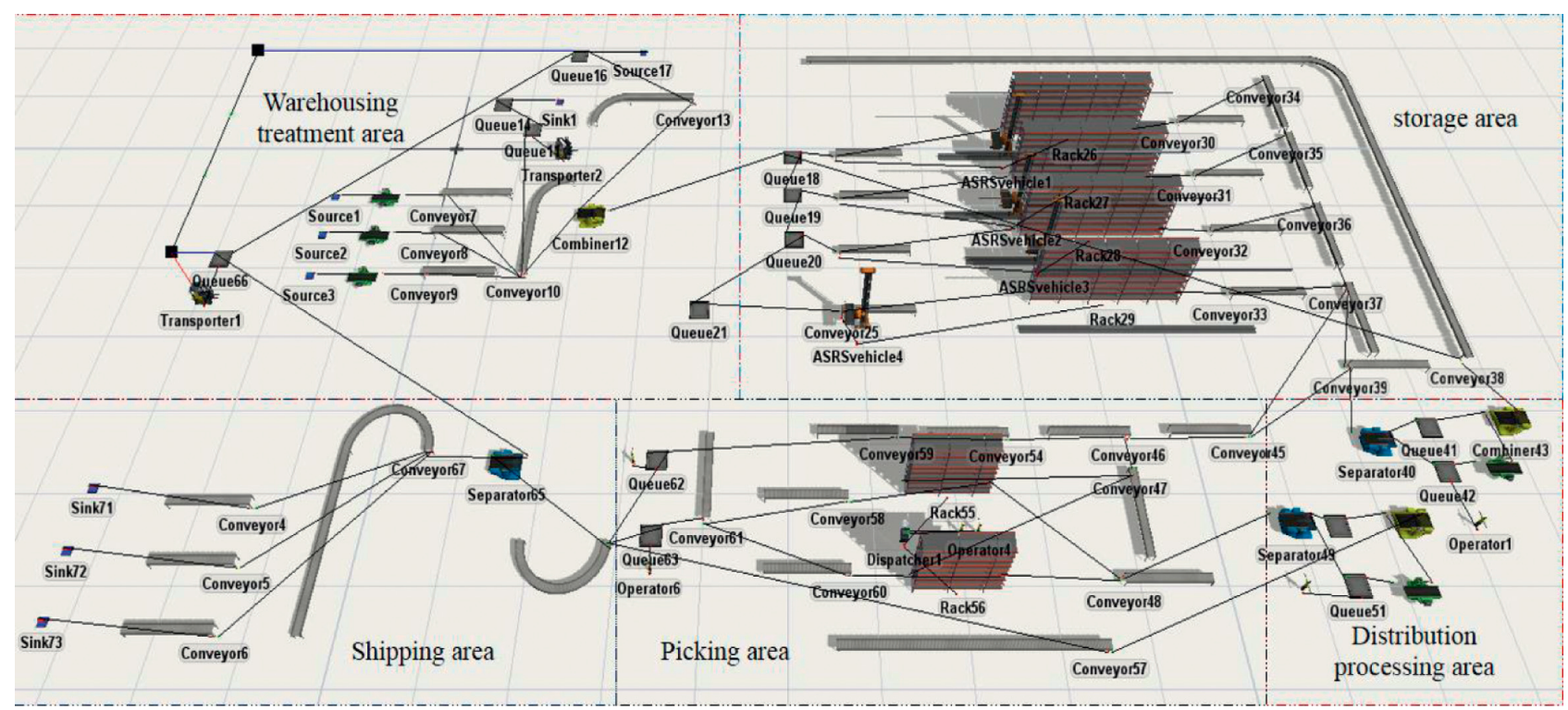

FIGURE 2: Overall concept operation diagram of the logistics distribution process.

3.2. Linkage Model of Basic Logistics Activities. According to the definition of the basic logistics activities, each operation in the warehousing treatment area is a basic logistics activity. The expression of the characteristic state equation for the connection between multiple basic logistics activities is introduced in stages as follows:

3.2.1. Material Supply Relationship. If the shipment material of a basic logistics activity (Activity 1) is another procurement item of basic logistics activity (Activity 2), there is a material supply relationship between Activity 1 and Activity 2, as shown in Figure 3(a). The start time, number of items, and logistics cost of Activity 2 are affected by the completion time, shipment quantity, and distribution cost of Activity 1. The above relationship can be expressed as a transformation relationship between the input feature state vector $x_{i 2}$ of the Activity 2 and the output feature state vector $x_{o 1}$ of the Activity 1 , as shown in Figure 3(b), with the characteristic state equation expressed as

$$
\mathbf{x}_{i 2}=\mathbf{I} \mathbf{x}_{o 1} \text {. }
$$

3.2.2. Device Sharing Relationship. If there are two basic logistics activities in the same work center in the order of scheduling, then there is a device sharing relationship between the two basic logistics activities, as shown in Figure 4(a).

Activity 2 needs to wait for Activity 1 to expire before the device is occupied, and the start time of Activity 2 is affected by the completion time of Activity 1 . The above relationship can be expressed as a transformation relationship $\mathrm{H}$ between the input feature state vector $x_{i 2}$ of the Activity 2 and the output feature state vector $x_{o 1}$ of the Activity 1 ; it is shown in Figure 4(b), with the characteristic state equation given as

$$
\mathbf{x}_{i 2}=\mathbf{H} \mathbf{x}_{o 1} \text {. }
$$

The transformation matrices $\mathbf{I}$ and $\mathbf{H}$ in equations (3) and (4) can be simplified into an instantaneous transition without object loss and cost effects, i.e.,

$$
\begin{gathered}
\mathbf{I}=\left[\begin{array}{lll}
1 & 0 & 0 \\
0 & 1 & 0 \\
0 & 0 & 1
\end{array}\right], \\
\mathbf{H}=\left[\begin{array}{lll}
1 & 0 & 0 \\
0 & 0 & 0 \\
0 & 0 & 0
\end{array}\right] .
\end{gathered}
$$

In formula (5), 1 indicates that there is an association relationship between the feature variables, but there is no 


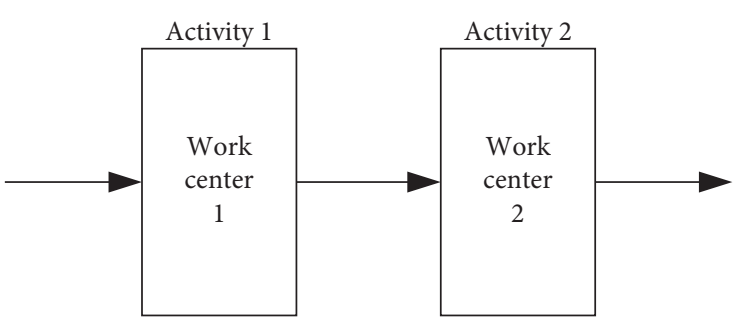

(a)

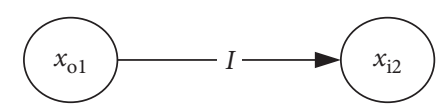

(b)

FIgURE 3: Material supply and characteristic state transformation.

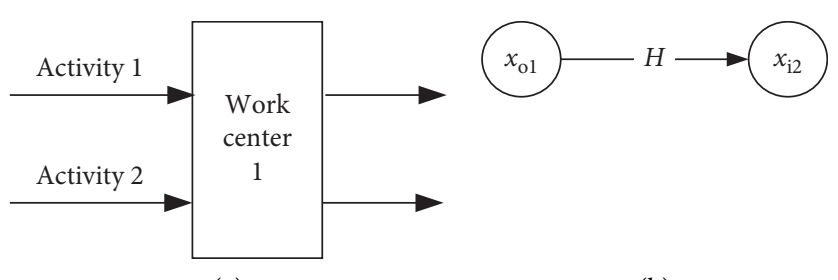

(a)

(b)

FIGURE 4: Device sharing and characteristic state transformation.

change in the physical quantity value and 0 indicates that there is no association relationship between the feature variables.

\section{Overall Logistics Distribution Process Model}

According to the two kinds of connection relationship between material supply and equipment sharing of basic logistics activities, the resource flow in the logistics distribution process can also be divided into two categories: material flow and information flow.

4.1. Characteristic State Transformation Process with Material Flow. The material flow process can be abstracted into a multistage state transformation process of the corresponding feature state vector. Taking object 1 in the logistics process shown in Figure 5 as an example. The object is processed by three work centers in sequence which are unloading, inspection, and transportation, to form a material flow, as shown in Figure 6(a). The process can be regarded as the six-stage state change process between the arrival of the object, the start of unloading, the end of unloading, the start of the inbound inspection, the end of the inbound inspection, the start of the transfer, and the end of the transfer, as shown in Figure 6(b).

Using the above method, the processing of each batch of materials in a complex logistics distribution process can be regarded as a material flow and can be expressed using a corresponding set of characteristic state equations.

4.2. Characteristic State Transition Process Accompanying Information Flow. In the logistics system, resources other than materials, including labor, facilities, and equipment, software information, and equipment wear, can be regarded as the medium for the transmission of various information

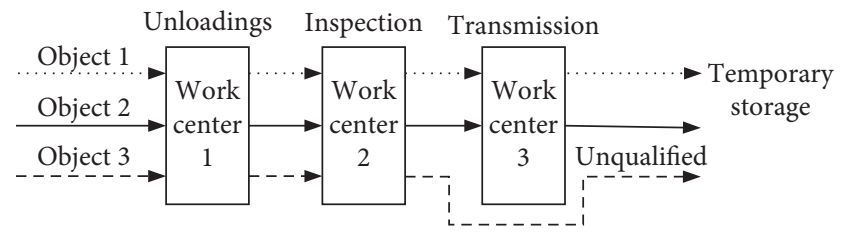

Figure 5: Process route of warehousing treatment area.

changes in the logistics and distribution process. Therefore, this resource flow is called information flow. The state of the information flow through each object batch is described using the feature state vector.

Take work center 1 in the logistics process shown in Figure 5 as an example. The work center sequentially processes object 1 , object 2 , and object 3 to form an information flow, as shown in Figure 7(a). The process can be regarded as a work center idle, and the processing of object 1 procedure 1 is started, end processing object 1 procedure 1 , start processing object 2 procedure 1 , end processing object 2 procedure 1 , start processing object 3 procedure 1 , and end processing object 3 procedure 1, i.e., a total of 7 states of 6 stage state change process as shown in Figure $7(\mathrm{~b})$.

Using the above method, the logistics process of each work center in the complex logistics distribution process can be regarded as a flow of information and can be expressed using a corresponding set of characteristic state equations.

4.3. Superposition of Feature State Transformation Process. Using the above method, various complex logistics processes can be divided into several material flows, energy flows, and time flows according to the material supply relationship and equipment sharing relationship. This information can then be expressed by corresponding characteristic state equations. Taking the logistics process shown in Figure 5 as an example, 


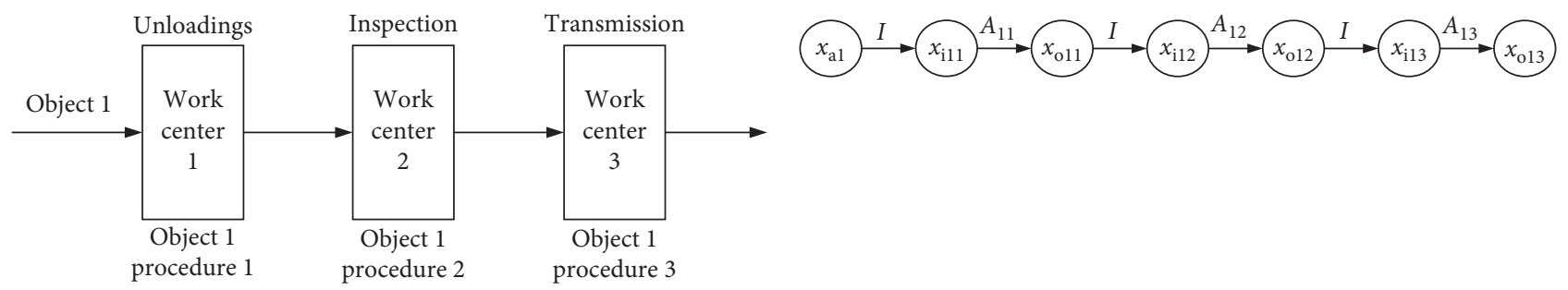

(a)

(b)

Figure 6: Material flow and characteristic state transformation.

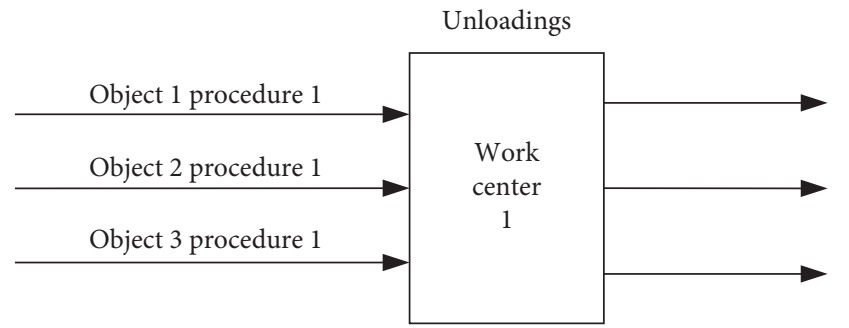

(a)

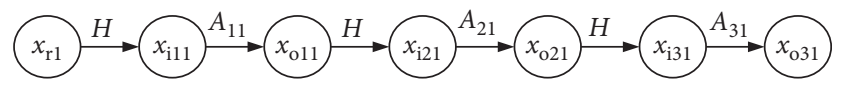

(b)

Figure 7: Information flow and characteristic state transformation.

this process uses three work centers to process three distribution batches of materials to form three energy flows and three material flows. The characteristic state transformation relationships of these material flows and energy flows are superimposed to form a characteristic state transformation expression for the entire material warehousing process, as shown in Figure 8.

When considering the characteristic state equations of basic logistics activities, multidimensional vectors $x_{i}$ and $x_{o}$ are used to represent the input and output feature vectors of the multistage logistics process, respectively. Shown in Figure 8 is the logistics distribution processes $x_{i}=\left(x_{a 1}\right.$, $\left.x_{a 2}, x_{a 3}, x_{r 1}, x_{r 2}, x_{r 3}\right)^{\mathrm{T}}$ and $x_{\mathrm{o}}=\left(x_{\mathrm{o} 13}, x_{\mathrm{o} 23}, x_{\mathrm{o} 32}\right)^{\mathrm{T}}$. The state change of each stage of material flow or information flow can be represented by a characteristic state equation. If the recurring feature state transformation is removed, the logistics process shown in Figure 8 can be expressed as a matrix equation composed of 24 characteristic state equations. This makes the equations easy to implement on a computer.

\section{Feature State Space}

5.1. Algebraic Operations of Characteristic Variables. Any complex logistics process can be abstracted into a set of state transition processes and can be described by a set of feature state equations. The $\otimes$ and $\oplus$ operations in the characteristic state equation correspond to the series and parallel combination relationships between multiple state transformations, respectively.

The characteristic variable operations in Table 1 can be performed by taking the maximum or minimum values in the sense of conventional algebra. In these operations, the $\oplus$ operation conforms to the commutative law and the conjunction law. The $\otimes$ operation conforms to the commutative law, the binding law, and the distribution law; the nature of these operations can also be extended to the matrix operation of $n \times n$ dimensions; the detailed operation can be found in the literature [13]. These two operators can be generalized to the matrix, and each type of matrix equation corresponds to a combination rule between basic logistics activities, as shown in Table 2 .

5.2. Feature State Space. A set of values in the logistics system may exist for all feature state vectors over a certain period. They have addition and multiplication operations, called the feature state space $\mathrm{C}$ of the logistics system.

Therefore, any state in a multistage complex logistics distribution process can be mapped to a point in the feature state space $\mathrm{C}$ since any basic logistics activity can be regarded as a transition between an input state and an output state. Therefore, the basic logistics activities are mapped to the directed line segments between the two points in the feature state space C. Furthermore, the multistage complex logistics distribution process formed by the superposition of multiple material flows and energy flows is mapped into multiple sections in the feature state space $\mathrm{C}$, continuously changing the path, as shown in Figure 9.

5.3. Critical Path and Bottleneck Analysis. For the time or quantity characteristic variable, because it follows the algebra operation rule of maximum or minimum addition, the influence of multiple change paths on the final state point in characteristic state space $\mathrm{C}$ can be expressed simply by one of the longest paths, which is usually called the critical path. The final delivery state of the object is determined by the critical path. At the same time, the final distribution state of 


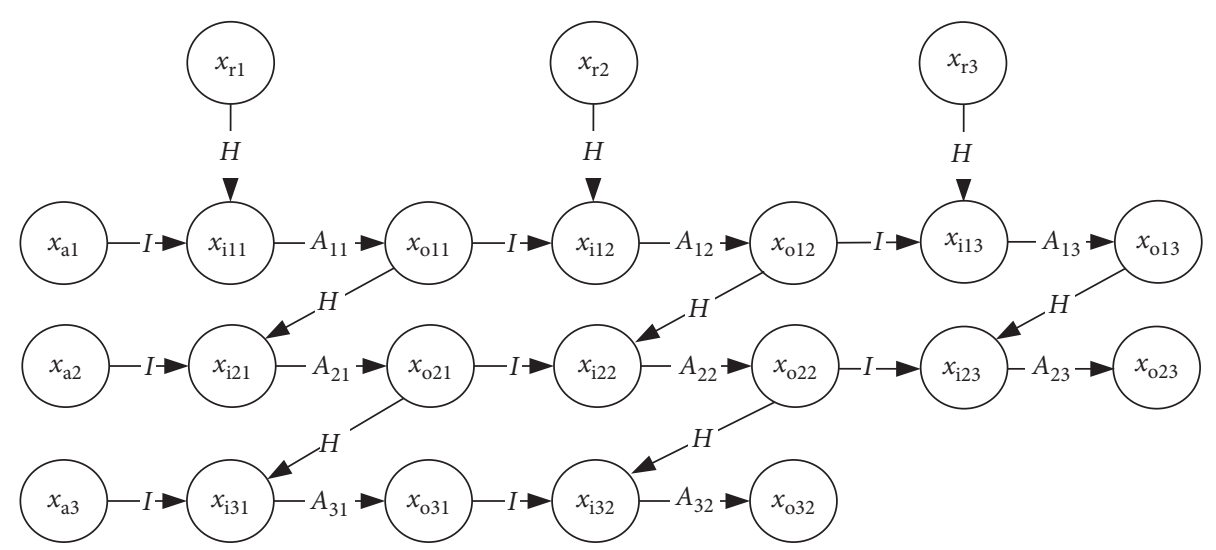

FigURE 8: Characteristic state transformation of modern logistics distribution process.

TABLE 1: Algebraic operation of characteristic state variables.

\begin{tabular}{lccc}
\hline Operation & Time & Quantity & Cost \\
\hline Multiplication $a \otimes b$ & $a+b$ & $a+b$ & $a+b$ \\
Addition $a \oplus b$ & $\max (a, b)$ & $\min (a, b)$ & $a+b$ \\
\hline
\end{tabular}

TABle 2: Matrix equations corresponding to the combination rules between the basic activities.

\begin{tabular}{lll}
\hline Combination rule & Transform graphics & Matrix equation \\
Series & & \\
Parallel-convergence relationship & &
\end{tabular}

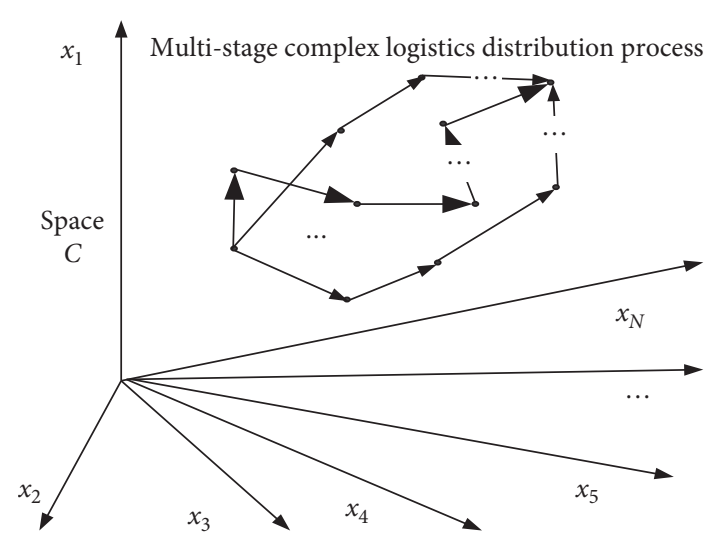

FIGURE 9: Expression of the logistics process in the feature state space C.

the object determines the objective function of the logistics control problem. Therefore, the extraction of the critical path can simplify the gradient analysis of the objective function in the logistics control problem.

In a complex logistics system, considering the uncertain factors such as order arrival, delivery time, and breakage rate, we can quantitatively analyze which resources have the greatest impact on the distribution cycle, object distribution rate, and logistics cost. This is called the bottleneck analysis of logistics system.

Firstly, we must define the symbol algebra rules that correspond to the maximal addition algebra. Let $a$ and $b$ be the symbols of the time characteristic variables and $v$ (a) and $v$ (b) be the values of the variables $a$ and $b$, respectively:

$$
\begin{array}{ll}
a \oplus b= \begin{cases}a, & v(a)>v(b), \\
b, & v(a)<v(b), \\
a \oplus b, & v(a)=v(b),\end{cases} \\
a \otimes b=a b .
\end{array}
$$

The above operation can be extended to the $n \times n$ dimensional matrix operation, and the symbolic expression of the serial relationship of the basic logistics activities are realized, thereby obtaining the critical path with the longest weighted length.

Secondly, we define the symbolic representation corresponding to the characteristic state equation of the logistics process. Taking the logistics distribution process 
shown in Figure 9 as an example, and given the arrival time of various objects in this logistics process, the release time of each work center and the specific values of the logistics activity processing time are shown in Table 3. The starting time of this logistics process is zero, and the other time is expressed by the relative time of the distance zero.

After the calculation, the critical path symbols for time characteristic variables are as follows:

$$
\begin{aligned}
& t_{o 13}=t_{p 13} t_{p 12} t_{p 11} t_{a 1}, \\
& t_{o 23}=t_{p 23} t_{p 22} t_{p 12} t_{p 11} t_{a 1}, \\
& t_{o 32}=t_{p 32} t_{p 22} t_{p 12} t_{p 11} t_{a 1} .
\end{aligned}
$$

Let $g_{\text {til }}$ be the reduction or increase of the distribution period of the $i$-th batch of objects after the work center $l$ adjusts the resources to improve the logistics efficiency by $1 \%$ according to the work center used by each basic logistics activity. The characteristic variable in Formulas (7)-(9) is replaced with the corresponding work center, and the value of each $g_{\text {til }}$ can be calculated. As shown in Table $4, g_{\text {til }}$ is a quantitative evaluation of the time bottleneck of the logistics system. It is also a manifestation of the objective function gradient in the logistics cycle.

It can be seen from Table 4 that the small change in the efficiency of logistics resources will affect the change of the distribution period of the object. The most influential work center is the inspection time of the logistics system. Through the same method, the replacement relationship between the quantity and cost characteristic variables can be calculated. The quantity and cost bottlenecks were measured with $g_{\mathrm{qi}}$ and $g_{\text {cil }}$, respectively.

\section{Optimization Model and Algorithm}

6.1. Logistics Control Problem Optimization Model. In the order-based distribution mode, the logistics system is in the distribution process of a logistics control cycle and the external input state $x_{i}$ of the system is determined by the market demand. For different logistics control schemes $s$, the logistics system has different delivery processes $A(s)$, which ultimately result in different object delivery statuses $x_{o}(s)$. Let the ideal completion status of each batch of objects be $x_{o}^{*}$. Under normal circumstances, the ideal object completion time is the customer's specified delivery date, and the corresponding time characteristic variable in $x_{o}^{*}$ is recorded as the delivery date; the ideal object distribution rate is $100 \%$ and is the corresponding quantitative feature in $x_{o}^{*}$. The variable is recorded as the number of objects required by the order; the direct cost is reduced as much as possible, and the corresponding cost characteristic variable in $x_{o}^{*}$ can be directly recorded as 0 . The line vector $w$ of the same dimension as $x_{o}(s)$ is used to express the unit penalty weight when the actual output state and the ideal state have an error. For the logistics control scheme $s$, these costs are recorded as $r(s)$. In summary, the optimization model of logistics control problems can be expressed as

$$
\begin{array}{ll}
\min , & z=\mathbf{w}\left(\mathbf{x}_{\mathrm{o}}(\mathbf{s})-\mathbf{x}_{\mathrm{o}}^{*}\right)^{+}+r(\mathbf{s}), \\
\text { subject to } & \mathbf{s} \in S .
\end{array}
$$

The $(\cdot)^{+}$in the formula $(10)$ represents $\max (\cdot, 0)$. In a complex logistics distribution process, the allocation of various resources has mutual influence. Therefore, the comprehensive logistics control problem of the system considering the multiple resources has become a complex optimization problem.

The characteristic state equation is used to realize the expression of the logistics process in the feature state space $C$ and is mapped to the decision space $D$. The distribution performance of different logistics systems under all possible logistics control schemes is composed of several discrete points in the decision space $D$. The abovementioned discrete point cloud is interpolated to obtain a three-dimensional surface with complex shapes, which is called the solution surface of the logistics control problem. The distribution of the two work centers in the actual example of Figure 8 and Table 3 considering, for example, the effect of the change on the overall performance of the logistics system (weighted delivery delay penalty and direct cost), is obtained by solving the three-dimensional space. As shown in Figure 10 , it can be seen that there is a certain continuity of the solution surface; the surface has certain monotonicity in the region far from the optimal solution; the solution surface has more complex fluctuations near the optimal solution. Combined with the gradient of the feature state space theory, the gradient search strategy will increase the speed or quality of the solution. This explains why the proposed algorithm can quickly find the approximate optimal solution under the guidance of the gradient.

6.2. Gradient-Based Genetic Algorithm (GBGA). The purpose of logistics control is to eliminate the system bottleneck and improve the system performance. The gradient guidance of the objective function is very important for the convergence of the accelerated optimization algorithm and for the speed control. We integrate the gradient search strategy into the mutation operator of the genetic algorithm [14], propose the bottleneck analysis based on the characteristic state space theory, and obtain the fast analysis method of the objective function gradient of the logistics control problem. This new algorithm is called the gradient-based genetic algorithm. The algorithm steps are as follows:

Step 1. Use the decision variable $s$ to encode the logistics control scheme; define the initial population size pop size; set $t=0$; randomly generate the initial population $s(0)=$ 
TABLe 3: Time characteristic variables in the logistics process.

\begin{tabular}{|c|c|c|c|c|c|c|c|}
\hline \multicolumn{4}{|c|}{ Basic logistics activity processing time (min) } & \multicolumn{2}{|c|}{$\begin{array}{c}\text { Raw material arrival } \\
\text { time (min) }\end{array}$} & \multicolumn{2}{|c|}{$\begin{array}{c}\text { Work center release } \\
\text { time (min) }\end{array}$} \\
\hline$t_{p 11}$ & 25 & $t_{p 22}$ & 35 & $t_{a 1}$ & 25 & $t_{r 1}$ & 20 \\
\hline$t_{p 12}$ & 50 & $t_{p 23}$ & 12 & $t_{a 2}$ & 30 & $t_{r 2}$ & 25 \\
\hline$t_{p 13}$ & 15 & $t_{p 31}$ & 26 & $t_{a 3}$ & 28 & $t_{r 3}$ & 15 \\
\hline$t_{p 21}$ & 22 & $t_{p 32}$ & 13 & - & - & - & - \\
\hline
\end{tabular}

TABLE 4: Time bottleneck analysis in the logistics process.

\begin{tabular}{lccc}
\hline Work center & Object delivery batch 1 & Item delivery lot 2 & Item delivery lot 3 \\
\hline 1 & $g_{t 11}=25 \times 1 \%=0.25$ & $g_{t 21}=25 \times 1 \%=0.25$ & $g_{t 31}=25 \times 1 \%=0.25$ \\
2 & $g_{t 12}=50 \times 1 \%=0.5$ & $g_{t 22}=g_{t 32}=(35+50) \times 1 \%=0.85$ \\
3 & $g_{t 13}=15 \times 1 \%=0.15$ & $g_{t 23}=12 \times 1 \%=0.12$ & $g_{t 32}=13 \times 1 \%=0.13$ \\
\hline
\end{tabular}

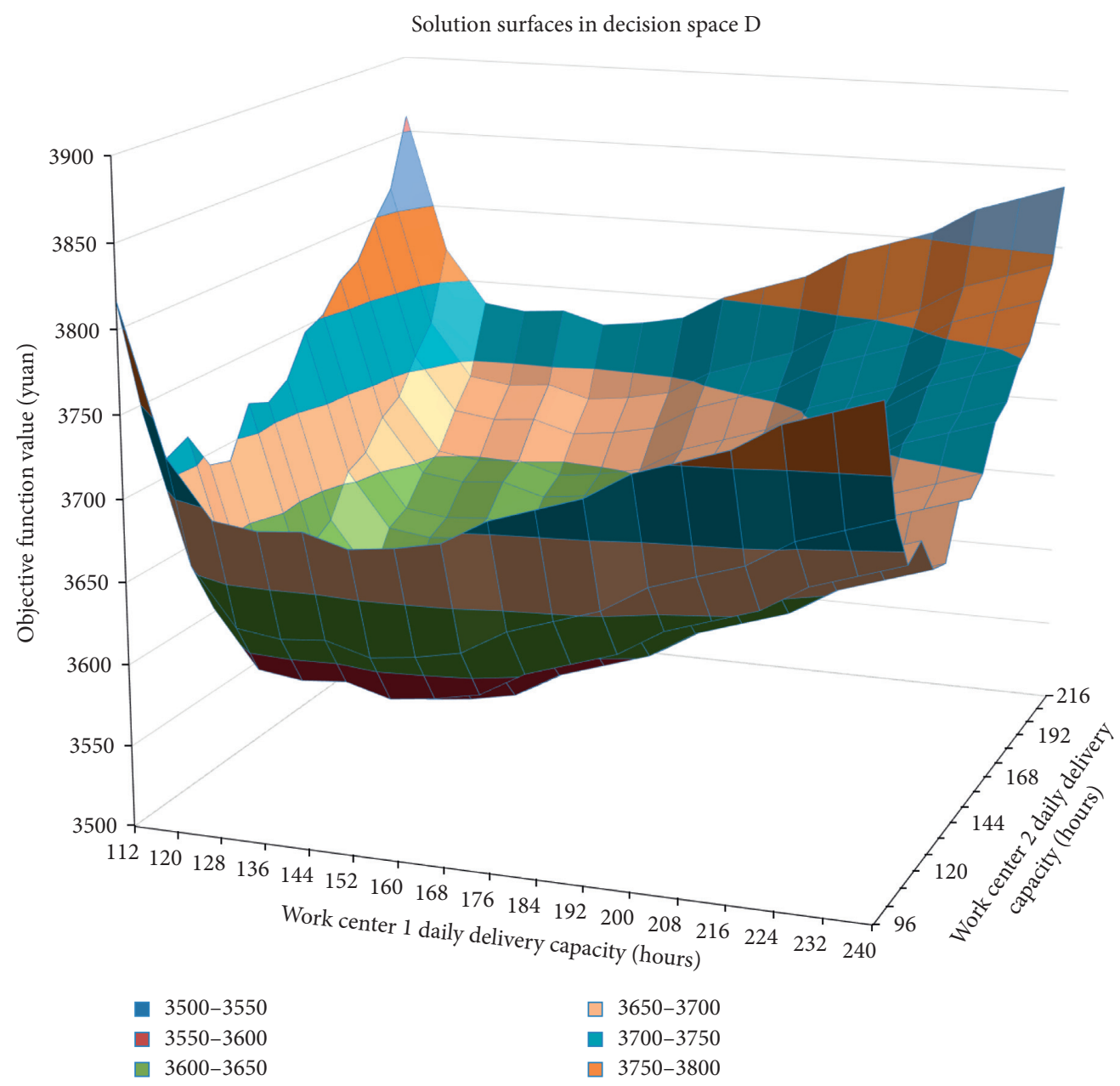

FIGURE 10: Solution surfaces of the decision space D

$\left(s_{1}, s_{2}, \ldots, s_{\text {pop_size }}\right)$ as the current population $s(t)$; and set the maximum iteration number limit $n_{g}$ and the freeze limit $\beta$.

Step 2. The gradient $G$ of the objective function is calculated by using the characteristic state equation established in synchronization with the simulation logistics process. Taking into account the logistics control problems of multiple optimization objectives of time, quality, and cost, the above calculated $g_{\mathrm{til}}, g_{\mathrm{qi}}$, and $g_{\text {cil }}(i=1,2, \ldots, n ; l=1,2, \ldots$, $m$ ) result in the matrix form: 


$$
\begin{aligned}
\mathbf{G}_{i l} & =\left[\begin{array}{ccc}
g_{\text {til }} & 0 & 0 \\
0 & g_{\text {qil }} & 0 \\
0 & 0 & g_{\text {cil }}
\end{array}\right], \\
\mathbf{G} & =\left[\begin{array}{cccc}
\mathbf{G}_{11} & \mathbf{G}_{12} & \cdots & \mathbf{G}_{1 m} \\
\mathbf{G}_{21} & \mathbf{G}_{22} & \cdots & \mathbf{G}_{2 m} \\
\ldots & \cdots & \cdots & \cdots \\
\mathbf{G}_{n 1} & \mathbf{G}_{n 2} & \cdots & \mathbf{G}_{n m}
\end{array}\right] .
\end{aligned}
$$

Step 3. The mutation operator selects a new solution $s_{1}$ from the neighborhood of the current solution $s_{0}$ obtained after the crossover operation. Let the current solution $s_{0}$ have $\mathrm{N}$ adjacent solutions. For any adjacent solution $s_{0 i}=(i=1,2$, $\ldots, N)$, let $\Delta s_{i}=s_{1}-s_{0 i}$, and calculate $\Delta z_{i}=w G \Delta s_{i}$; The row vector $w$ is the weight value of multiple objective functions. Let $\Delta z_{\max }$ be the maximum value in $\Delta z_{i}(i=1,2, \ldots, N)$, and select the new solution $s_{1}$ from the adjacent solutions of the current solution $s$ according to the following probability:

$$
P\left(\mathbf{s}_{1}=\mathbf{s}_{0 i}\right)=\frac{\left(\Delta z_{\max }-\Delta z\left(\mathbf{s}_{0 i}\right)\right)^{\gamma}}{\sum_{i=1}^{N}\left(\Delta z_{\max }-\Delta z\left(\mathbf{s}_{0 i}\right)\right)^{\gamma}} .
$$

Thus, the adjacent solutions that reduce the value of the objective function are more likely to have a higher probability of selection. The parameter $\gamma$ in the equation (12) is used to adjust the degree of influence of the gradient of the objective function on the search direction, which is called the guidance rate.

Step 4. $t=t+1$ determines whether the iteration reaches the termination condition. If it has been reached, end the search and use the best individual in the current population as the final optimization result; otherwise, go to step 2. The algorithm has two termination conditions: one is that the total number of iterations exceeds the maximum number of iterations $n_{g}$; the other is that there is no better quality individual in the middle and long terms, and the corresponding number of consecutive iterations exceeds the freeze limit $\beta$.

The improvement of the traditional genetic algorithm based on the above hybrid algorithm mainly exists in the mutation operator. The gradient analysis result obtained by the feature state space theory is used to replace the random mutation strategy in the traditional genetic algorithm, in order to obtain a higher convergence speed.

\section{Case Verification}

The feature state space theory and the method proposed in this paper are used to solve the logistics control problem of actual logistics distribution enterprises. From the perspective of testing the logistics process model accuracy, bottleneck analysis method performance, and logistics control algorithm performance, the feasibility and effectiveness of the proposed theory and method are verified and compared with traditional methods.
7.1. Background and Basic Data. We take a modern pharmaceutical logistics center at a listed pharmaceutical company as an example. The logistics center distributes medicines according to the order and extracts the logistics and distribution data of the enterprise from January to December 2018 in the logistics management system. There are two types of objects (product series) expressed as object series 1 and series 2 . As shown in Tables 5-7, we can get the basic information, logistics process, and statistical characteristics of the work center and staffing.

The scheduling rule adopted by the logistics distribution center is the earliest scheduled delivery date first (EDD) and the first come first process (FCFS) when it is equal to the delivery date. The number of facilities and equipment, the number of personnel, and the direct cost per unit time of each work center are shown in Table 5.

The collected historical data are classified according to the object series. The average and standard deviation of order arrival interval, order batch, and lead time of each item series in each quarter are given in Table 6 . The average values of delay penalty and damage penalty in different quarters are also given in Table 6 . The statistical values of object handling time, damage rate, and unit logistics cost are given in Table 7. The order arrival interval, order batch, order lead time, and operation handling time are generated by corresponding normal distribution. The delay penalty and unqualified penalty are directly fixed by historical average.

\subsection{Experimental Calculations and Results}

\subsubsection{Testing the Accuracy of the Logistics Process Model.} The weighted deferred penalty, weighted nonconforming product penalty, and inventory cost of Table 6 are optimized. The simulation results of the logistics process model for each quarter in 2018 are compared with the actual logistics record data. The results are shown in Table 8 .

According to the calculation results, the maximum error between the simulated data and the actual data in Table 8 is $-2.39 \%$. This is mainly due to lack of sufficient historical samples; errors caused by uncertainty in the data collection and generation; some jobs are cancelled or frozen by customers or some tasks are not scheduled according to certain rules. However, the distribution of these errors is stable between $-2.02 \%$ and $-2.39 \%$. As the logistics management system continues to increase in operation time, using more historical data, it is possible to consider subtracting a historical error from the simulation results of the logistics process. This will further improve the simulation accuracy of the model.

7.2.2. Test Objective Function Gradient Analysis Method. The average value of the difference between the gradient analysis method and the pure simulation method in the different work centers or the adjustment of the target function after adjusting for different key materials is referred to as the average error. This indicator is used to express the accuracy of the gradient analysis method compared to the pure simulation method. Through the logistics control, 
TABLE 5: Equipment and personnel information.

\begin{tabular}{lccc}
\hline Device name (model) & Equipment quantity & Configured number of people & Direct cost (¥/hour) \\
\hline Freight type agv transport trolley & 1 & 1 & 5.74 \\
Forklift & 2 & 2 & 8.7 \\
Conveyor sorting system & 4 & 8 & 8.14 \\
Intelligent picking trolley & 2 & 2 & 5.21 \\
Stacker & 4 & 4 & 8.93 \\
Mops & 8 & 8 & 3.56 \\
Mobile shelf & 2 & 2 & 1.91 \\
High shelf & 4 & 4 & 1.32 \\
Standard tray & 340 & 2 & 1.21 \\
Bar code scanner & 10 & 5 & 2.56 \\
Pda acquisition device & 8 & 8 & 2.64 \\
\hline
\end{tabular}

TABLE 6: Basic information of two series of delivered objects.

\begin{tabular}{|c|c|c|c|c|c|c|c|c|c|}
\hline \multirow{2}{*}{ Time } & \multirow{2}{*}{$\begin{array}{l}\text { Object } \\
\text { Series }\end{array}$} & \multicolumn{2}{|c|}{$\begin{array}{l}\text { Order arrival interval } \\
\text { (days) }\end{array}$} & \multicolumn{2}{|c|}{ Order batch (unit) } & \multicolumn{2}{|c|}{ Order lead time (days) } & \multirow{2}{*}{$\begin{array}{l}\text { Deferred penalty } \\
\text { (¥/day) }\end{array}$} & \multirow{2}{*}{$\begin{array}{l}\text { Damaged item } \\
\text { penalty (¥/day) }\end{array}$} \\
\hline & & Mean & $\begin{array}{l}\text { Standard } \\
\text { deviation }\end{array}$ & Mean & $\begin{array}{l}\text { Standard } \\
\text { deviation }\end{array}$ & Mean & $\begin{array}{l}\text { Standard } \\
\text { deviation }\end{array}$ & & \\
\hline \multirow{2}{*}{$\begin{array}{l}\text { First } \\
\text { quarter }\end{array}$} & 1 & 12.76 & 4.22 & 12.57 & 8.24 & 25.52 & 1.03 & 220 & 2361 \\
\hline & 2 & 14.94 & 7.26 & 12.45 & 7.79 & 25 & 1.62 & 211 & 2124 \\
\hline \multirow{2}{*}{$\begin{array}{l}\text { Second } \\
\text { quarter }\end{array}$} & 1 & 17.68 & 6.41 & 10.24 & 6.86 & 26.77 & 1.1 & 165 & 1960 \\
\hline & 2 & 20.71 & 0.99 & 10.1 & 6.78 & 26.35 & 1.52 & 152 & 1896 \\
\hline \multirow{2}{*}{$\begin{array}{l}\text { Third } \\
\text { quarter }\end{array}$} & 1 & 11.85 & 4.38 & 11.34 & 5.21 & 25.97 & 1.81 & 85 & 1127 \\
\hline & 2 & 13.89 & 2.1 & 11.08 & 5.12 & 25.11 & 1.26 & 301 & 4213 \\
\hline \multirow{2}{*}{$\begin{array}{l}\text { Fourth } \\
\text { quarter }\end{array}$} & 1 & 8.85 & 2.6 & 15.39 & 9.89 & 25.94 & 1.19 & 284 & 3765 \\
\hline & 2 & 10.37 & 3.88 & 15.21 & 9.23 & 25.69 & 1.63 & 230 & 2651 \\
\hline
\end{tabular}

TABLE 7: Single-piece processing time, breakage rate, and logistics cost for each process.

\begin{tabular}{|c|c|c|c|c|c|c|c|c|}
\hline \multirow{3}{*}{$\begin{array}{l}\text { Name } \\
\text { Process }\end{array}$} & \multicolumn{4}{|c|}{ Object 1} & \multicolumn{4}{|c|}{ Object 2} \\
\hline & \multicolumn{2}{|c|}{$\begin{array}{l}\text { Single processing time } \\
\text { (minutes) }\end{array}$} & \multirow{2}{*}{$\begin{array}{c}\text { Damage rate } \\
(\%)\end{array}$} & \multirow{2}{*}{$\begin{array}{l}\text { Unit logistics cost } \\
\text { (Yuan) }\end{array}$} & \multicolumn{2}{|c|}{$\begin{array}{l}\text { Single processing time } \\
\text { (minutes) }\end{array}$} & \multirow{2}{*}{$\begin{array}{c}\text { Damage rate } \\
(\%)\end{array}$} & \multirow{2}{*}{$\begin{array}{l}\text { Unit logistics cost } \\
\text { (Yuan) }\end{array}$} \\
\hline & Mean & $\begin{array}{l}\text { Standard } \\
\text { deviation }\end{array}$ & & & Mean & $\begin{array}{l}\text { Standard } \\
\text { deviation }\end{array}$ & & \\
\hline$j=1$ & 4.08 & 0.12 & 0.003 & 15.1 & 3.81 & 0.57 & 0.027 & 8.4 \\
\hline$j=2$ & 3.68 & 0.59 & 0.006 & 15.8 & 3.99 & 0.77 & 0.006 & 17.5 \\
\hline$j=3$ & 0.96 & 0.2 & 0.009 & 3.1 & 1.01 & 0.22 & 0.009 & 2.7 \\
\hline$j=4$ & 3.4 & 0.82 & 0.023 & 12.6 & 3.35 & 0.73 & 0.024 & 18 \\
\hline$j=5$ & 9.8 & 0.41 & 0.009 & 20.1 & 9.74 & 0.87 & 0.09 & 17.4 \\
\hline$j=6$ & 4.12 & 0.92 & 0.004 & 17.7 & 3.94 & 0.05 & 0.034 & 18.7 \\
\hline$j=7$ & 1.24 & 0.04 & 0.013 & 4 & 1.25 & 0.22 & 0.001 & 3.2 \\
\hline$j=8$ & 4.6 & 0.2 & 0.008 & 19.8 & 4.5 & 1.21 & 0.09 & 18.9 \\
\hline$j=9$ & 6.88 & 0.31 & 0.05 & 63.5 & 6.58 & 0.48 & 0.05 & 22 \\
\hline$j=10$ & 1.12 & 0.31 & 0.012 & 3.6 & 1.23 & 0.33 & 0.013 & 10 \\
\hline$j=11$ & 9.36 & 2.22 & 0.08 & 19.7 & 8.76 & 2.43 & 0.07 & 2.7 \\
\hline$j=12$ & 13.46 & 0.34 & 0.011 & 28.6 & 13.03 & 3.89 & 0.001 & 10.8 \\
\hline
\end{tabular}

TABLE 8: Simulation accuracy of the logistics process model.

\begin{tabular}{lccc}
\hline \multirow{2}{*}{ Simulation period } & \multicolumn{2}{c}{ Target function value (¥) } & Actual record data \\
& Simulation result data & 4319708 & Error (\%) \\
\hline First quarter (1-3 months) & 4223467 & 3973536 & -2.23 \\
Second quarter (4-6 months) & 3884675 & 4687902 & -2.24 \\
Third quarter (7-9 months) & 4575676 & 4328744 & -2.39 \\
Fourth quarter (10-12 months) & 4239432 & -2.06 \\
\hline
\end{tabular}


TABle 9: Average error after changing work center distribution capacity and material stock.

\begin{tabular}{lccc}
\hline Distribution capacity (\%) & Average error (\%) & Safety stock (\%) & Average error (\%) \\
\hline-5 & -4.04 & -20 & -3.45 \\
-10 & -3.57 & -10 & -3.61 \\
5 & 3.68 & 10 & 3.16 \\
10 & 4.32 & 20 & 4.08 \\
15 & 6.84 & 30 & 5.4 \\
\hline
\end{tabular}

TABle 10: Control parameter settings for the test algorithm.

\begin{tabular}{lcc}
\hline Algorithm number & Algorithm type & Control parameter setting \\
\hline 1 & GA & pop_size $=5 ; p_{c}=0.3 ; p_{m}=0.1 ; n_{g}=330$. \\
2 & GA & pop_size $=3 ; p_{c}=0.5 ; p_{m}=0.3 ; n_{g}=70$. \\
3 & GBGA & pop_size $=5 ; p_{c}=0.3 ; p_{m}=0.1 ; n_{g}=330$. \\
4 & GBGA & pop_size $=3 ; p_{c}=0.5 ; p_{m}=0.3 ; n_{g}=70$. \\
\hline
\end{tabular}

the distribution ability of different work centers is changed by $-10 \%,-5 \%,+5 \%,+10 \%$, and $+15 \%$, respectively. Table 9 shows the calculation results in the case when the inventory of different key materials changes by $-20 \%,-10 \%,+10 \%$, $+20 \%$, and $+30 \%$.

According to the above calculation results, when the distribution capacity of the work center is small $( \pm 10 \%)$ and the inventory change of key materials is small $( \pm 20 \%)$, the gradient analysis method and the pure simulation method have very close objective function calculation results (the average error is less than 5\%). Due to the actual logistics control problem, the ability of the adjacent optional control scheme to adjust the capacity of the work center does not exceed \pm 10 percent and the inventory adjustment of key materials does not exceed \pm 20 percent. Therefore, when solving practical engineering problems, the gradient analysis method can replace the pure simulation method with approximate results.

The biggest advantage of using the gradient analysis method is its fast calculation response. For a logistics control problem with an adjustable work center and a critical material quantity $N$, the calculation time of the gradient analysis method is theoretically only $1 / 2 \mathrm{~N}$ of the pure simulation method.

\subsubsection{Preferred Algorithm for Testing Logistics Control} Scheme. The integrated gradient search strategy genetic algorithm (GBGA) based on feature state space theory is compared with the traditional neighborhood search genetic algorithm (GA) [14]. Two groups of control parameters with better effect are selected for the test. The parameter settings are shown in Table 10.

Each algorithm of each calculation repeats 5 times. For the multiple repeated optimization results of the same calculation of the same algorithm, the maximum value, minimum value, average value of the objective function, and average value of the single optimization run time are as shown in Table 11.

According to the above calculation results, the following conclusions can be drawn:
(1) The GBGA algorithm can find a scheme with higher quality than the GA algorithm, and the solution quality is more stable. The average value of the objective function obtained by the GBGA algorithm is 9.71\% lower than the GA algorithm; the difference between the maximum and minimum values of the objective function is only $21.34 \%$ of the GA algorithm.

(2) The solution speed of GBGA algorithm is obviously better than GA algorithm. The average search time is only $24.35 \%$ of GA algorithm.

(3) Compared with the gradient search strategy, different algorithm control parameters have much less influence on the quality of the algorithm. For the GBGA and GA algorithms, the difference in solution quality between the two different parameter settings is only $7.21 \%$.

7.3. Practical Application for Logistics Enterprises. The change of market demand in the logistics industry will lead to the imbalance of resource supply and demand. Through the characteristic state equation model, this phenomenon appears in the feature space as the imbalance of the gradient of the flow control objective function in various resource optimization adjustment directions. Using the gradient imbalance that can be quantitatively described mathematically, companies can learn which resources have the greatest impact on the overall performance of the logistics system and what the specific impact is to achieve optimal tuning:

7.3.1. Analysis of Bottlenecks in Distribution Capacity. In the modern logistics distribution system, the work center refers to the facilities and equipment with personnel as the core. Whether a work center reaches the distribution capacity bottleneck to increase/decrease the distribution capacity of the work center unit and the performance change of the logistics system (average delivery cycle/breakage rate/logistics cost) is evaluated. After the daily distribution capacity of $+10 \%$ of each work center of Tables 5 and 11, respectively, the shortening of the average delivery period of the object 
TABLE 11: Objective function values solved by each algorithm.

\begin{tabular}{lcccc}
\hline Name & & Target function value (1000¥) & Maximum & Operation time (seconds) \\
& Average value & Minimum value & 1584 & 7520 \\
GA & 1527 & 1521 & 1610 & 1607 \\
GA & 1536 & 1521 & 1521 & 1073 \\
GBGA & 1521 & 1521 & 1529 & 733 \\
\hline
\end{tabular}

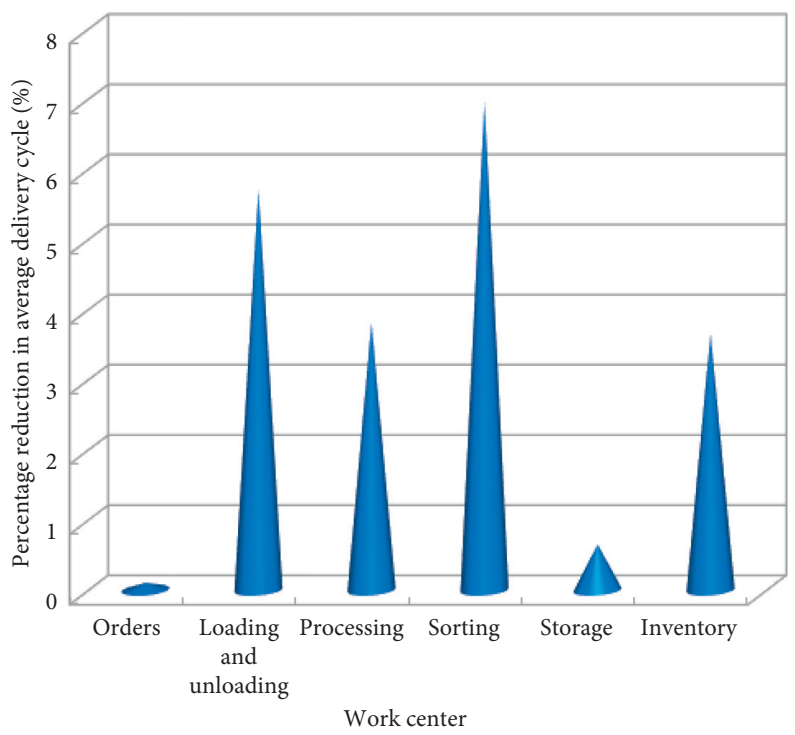

FIGURE 11: Bottleneck analysis of the distribution capacity in the logistics system.

TABLE 12: Simulation accuracy of the logistics process model.

\begin{tabular}{lccccccccc}
\hline & product & \multicolumn{3}{c}{ The $k$ th raw material } & \multicolumn{4}{c}{ Parts or products after the $j$ th process } \\
& Series & 1 & 2 & 3 & 4 & 1 & 2 & 3 & 4 \\
\hline \multirow{2}{*}{ Safety stock } & 1 & 30 & 30 & 30 & 30 & - & 10 & - & 10 \\
\multirow{3}{*}{ Cost $(¥)$} & 2 & 30 & 30 & 30 & 30 & - & 10 & - & 10 \\
& 1 & 245 & 310 & 421 & 105 & - & 879 & - & 986 \\
& 2 & 245 & 310 & 421 & 87 & - & 852 & - & 934 \\
\hline
\end{tabular}



ZZZ Inventory cost

$\because$ Delay in delivery

- - Variable increase

FIGURE 12: Bottleneck analysis of delivery delay and inventory cost. 
accounts for the percentage of the original delivery cycle as shown in Figure 11.

7.3.2. Analysis of Material Supply Bottlenecks. The performance change (average distribution cycle/breakage rate/ logistics cost) of the logistics system is evaluated after a certain material reaches the bottleneck to increase/decrease the safety stock quantity per unit of the material. The decrease in the average delivery delay time in the data from Tables 5 and 12 and the increase in the monthly inventory cost of the corresponding portion are shown in Figure 12.

The above two types of bottleneck analysis can be used to adjust the safety stock of key materials. Under the condition of limited inventory space and purchasing funds, this kind of adjustment usually involves multiple materials. It is possible to optimize the search strategy by using the feature state space model to find the optimal result. The logistics control plan can provide the following decision support for logistics enterprise managers:

(1) It is suggested that in the future technological transformation, the enterprise managers should give priority to preparing the best equipment, tools, and personnel with composite labor skills for the work centers that are easy to become the bottleneck of distribution capacity. Prepare more suitable inventory space for the objects that have the potential to become the bottleneck of material supply.

(2) Provide guidance for business managers to optimize the allocation of equipment and personnel according to market changes and optimize the setting of safety stocks.

(3) Provide direction guidance for the future investment of enterprise managers, that is, when expanding the distribution capacity in the future, prioritize the increase of equipment in the work center that eliminates the bottleneck, and prioritize the expansion of the material warehouse to avoid the bottleneck.

\section{Conclusion}

(1) A characteristic state equation model that can describe the multistage multiproduct distribution process of typical logistics distribution enterprises has been constructed. From the point of view of the feature information state, the mathematical description of time, quality, cost, and other feature information changes in the distribution process is realized. It is convenient to describe and analyze large-scale logistics process in the computer system.

(2) A feature state space model and method for bottleneck analysis of logistics distribution enterprises are proposed. The logistics distribution process is mapped onto the feature state space. The linear tangent plane equation is used to describe the local variation law of the solution surface, which provides a theoretical basis for the bottleneck analysis of the logistics system. Its computational efficiency is improved in algorithm complexity level compared with the traditional simulation method.

(3) The feature state space model and method for logistics control are proposed for the first time. The logistics control problem is transformed into the path-planning problem in the feature state space. The bottleneck analysis result of the logistics system is used as the objective function gradient of the logistics control problem. The integrated gradient search strategy enters the optimized search algorithm in the field to improve the solution speed and the quality of the logistics control problem.

\section{Data Availability}

No data were used to support this study.

\section{Conflicts of Interest}

The authors declare that they have no conflicts of interest.

\section{References}

[1] A. E. Rubtsov, E. V. Ushakova, and T. V. Chirkova, "Peculiarities of solving the problems of modern logistics in highrise construction and industrial production," in Proceedings of the International Scientific Conference on High-Rise Construction, HRC 2017, EDP Sciences, Samara, Russia, September 2017.

[2] C. S. Tang and L. P. Veelenturf, "The strategic role of logistics in the industry 4.0 era," Transportation Research Part E: Logistics and Transportation Review, vol. 129, pp. 1-11, 2019.

[3] R. Giusti, D. Manerba, G. Bruno, and R. Tadei, "Synchromodal logistics: an overview of critical success factors, enabling technologies, and open research issues," Transportation Research Part E: Logistics and Transportation Review, vol. 129, pp. 92-110, 2019.

[4] H. Khorshidian, M. Akbarpour Shirazi, and S. M. T. Fatemi Ghomi, "An intelligent truck scheduling and transportation planning optimization model for product portfolio in a crossdock," Journal of Intelligent Manufacturing, vol. 30, no. 1, pp. 163-184, 2019.

[5] L. Wieczorek and P. lgnaciuk, "Continuous genetic algorithms as intelligent assistance for resource distribution in logistic systems," Data, vol. 3, no. 4, p. 68, 2018.

[6] P.-Y. Hsu, P. Angeloudis, and M. Aurisicchio, "Optimal logistics planning for modular construction using two-stage stochastic programming," Automation in Construction, vol. 94, no. 1, pp. 47-61, 2018.

[7] L. Dymowa and P. Sevastjanov, "Fuzzy simulation and optimization of production and logistic systems," Studies in Fuzziness \& Soft Computing, Springer, Berlin, Germany, pp. 249-277, 2010.

[8] C. G. Gómez S., L. Cruz-Reyes, J. J. González B., H. J. Fraire H., R. A. Pazos R., and J. J. Martínez P., "Ant colony system with characterization-based heuristics for a bottled-products distribution logistics system," Journal of Computational and Applied Mathematics, vol. 259, no. part B, pp. 965-977, 2014.

[9] D. Wang, D. Zhang, and Y. Ma, "New approach to automated conceptual design of mechanical system by means of state- 
space," Chinese Journal of Mechanical Engineering, vol. 39, no. 3, pp. 22-27, 2003.

[10] L. Zhang and D. Wang, "New approach to automated conceptual design of hybrid mechanical system by means of characteristic state space," Chinese Journal of Mechanical Engineering, vol. 42, no. 12, pp. 26-35, 2006.

[11] W. Ma and D. Wang, "Energy characteristic state model of hydraulic system conceptual design," Journal of Mechanical Engineering, vol. 46, no. 4, pp. 129-135, 2010.

[12] X. Ma, J. Yu, and Y. Ren, Case Study of Modeling and Simulation of Flexsim Logistics System, Chemical Industry Press, Beijing, China, 2018.

[13] W. C. Chen, J. W. Yu, E. S. Qi, and W. Liu, "Match mechanism of physical and information processes in discrete series production system," Computer Integrated Manufacturing Systems, vol. 25, no. 11, pp. 2923-2934, 2018.

[14] Z. Cai and M. G. Liang, "An intra-street source routing mechanism based on genetic algorithm in VANETs," Journal of Transportation Systems Engineering and Information Technology, vol. 19, no. 4, pp. 43-49, 2019. 